\title{
A Prospective Study to Evaluate Causes and Outcomes of Acute Kidney Injury in Patients of Cirrhosis of Liver in a Tertiary Care Centre
}

Authors

\author{
Dr B. Ashok Babu ${ }^{1}$, Dr K.Vidyasagar ${ }^{2}$, Dr R.Ranganadha Rao ${ }^{3}$, \\ Dr M.Madhusudhana Babu ${ }^{4}$ \\ ${ }^{1}$ Postgraduate, Department of General Medicine, GEMS \\ ${ }^{2}$ Consultant Nephrologist, Department of General Medicine, GEMS \\ ${ }^{3}$ Senior Resident, Department of General Medicine, GEMS \\ ${ }^{4}$ Professor, Department of General Medicine, GEMS
}

\section{Introduction}

Acute kidney injury (AKI) frequently occurs in patients with cirrhosis and was associated with significant mortality. ${ }^{1,2}$ mortality in cirrhosis has been shown to increase progressively in association with an increase in the severity of $\mathrm{AKI}^{2}$ The most common causes of AKI in cirrhosis are pre-renal azotemia (volumeresponsive pre-renal $\mathrm{AKI}$ ), acute tubular necrosis (ATN) and hepatorenal syndrome (HRS), a functional type of pre-renal AKI exclusive of cirrhosis that does not respond to volume repletion. Recent studies suggest using the Acute Kidney Injury Network (AKIN) definition of AKI, which closely correlates with mortality in cirrhosis.

\section{Aim of the Study}

- To study the incidence of different patterns of AKI in cirrhosis.

- To evaluate the impact of AKI severity on patient survival

\section{Materials \& Methods}

This was a prospective observational study carried at GEMS hospital, department of General medicine, Srikakulam, Andhra Pradesh from November 2017 to January 2019, and followup for three months. Patients with a diagnosis of cirrhosis of the liver were evaluated for the presence of AKI. All data was collected as part of the routine clinical care in hospitalized patients, which included demographics, medical history, laboratory findings like CBC, LFT, viral markers, ascitic fluid analysis, coagulation profile, cultures of blood, urine, ascitic fluid, radiology, procedural findings including medications

\section{Inclusion Criteria}

1) Age of the patient's $\geq 18$ years

2) Patients with a diagnosis of cirrhosis

3) Presence of AKI Based on AKIN criteria

\section{Exclusion Criteria}

1) Patients with less than 18 years of age

2) Chronic kidney disease

3) Patients with uncontrolled Diabetes, HTN, using nephrotoxic agents

4) Acute or chronic renal replacement therapy before admission 
In one year period, a total of 217 cirrhotic patients were identified, out of which 87 had different forms of renal disorders. After the exclusion of C.K.D and h/o structural abnormalities, a total of 80 patients with cirrhosis and renal failure were enrolled in the study over a period of 12 months.

\section{Statistical Analysis}

For descriptive statistical analysis, mean, standard deviation, and frequencies were calculated. Different characteristics were represented as

\section{Results}

In one year period total of 217 cirrhotic patients identified out of which 56 had different forms of AKI.

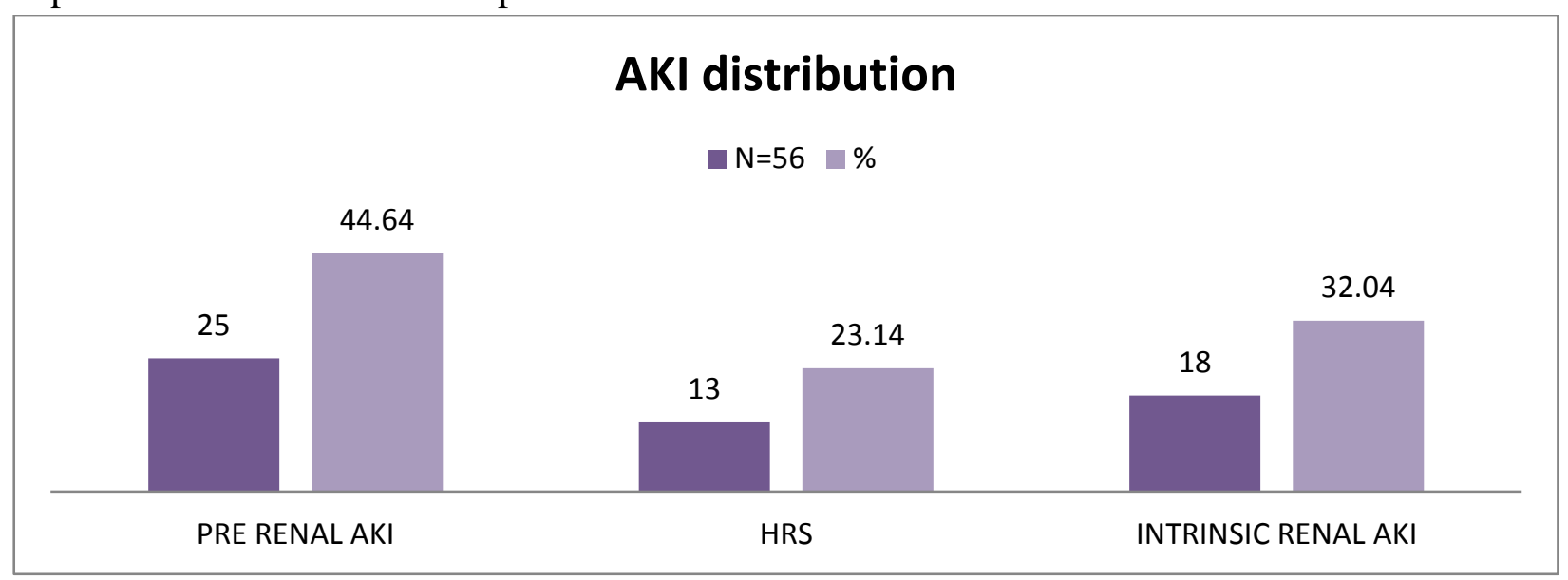

The spectrum of AKI in Patients with Cirrhosis of Liver (figure 1): The incidence of AKI in patients with cirrhosis of the liver in present study was $25.8 \%$ (56/217).Out of 56 numbers or percentages wherever required. Statistical analysis was done by statistical software SPSS for Windows v21.0.P-Value shows statistical significance when it was $<0.05$.

\section{Outcome}

Outcome was mortality during the hospitalization or follow up for 90 days.

\section{Stage wise distribution of AKI}

\begin{tabular}{|c|c|c|c|}
\hline AKIN stage & TOTAL & SURVIVORS & NON -SURVIVORS \\
\hline 1 & $8(14.3 \%)$ & $8(19.5 \%)$ & $0(0 \%)$ \\
\hline 2 & $17(30.4 \%)$ & $16(39.0 \%)$ & $1(6.7 \%)$ \\
\hline 3 & $31(55.4 \%)$ & $17(41.5 \%)$ & $14(93.33 \%)$ \\
\hline
\end{tabular}

AKIN stages 1,2 and 3 attained in 14.3\%, 30.4\% and $55.4 \%$ of patients respectively. Mortality increased in a stage response manner with the patients, the spectrum of AKI includes pre-renal (44.64\%) intrinsic renal (32.04\%) and HRS (23.14\%). Even though HRS is a type of pre-renal $\mathrm{AKI}$, it is not volume responsive.

Association of HRS with Mortality

\begin{tabular}{|l|c|c|c|}
\hline & HRS & NON HRS & TOTAL \\
\hline NON SURVIVORS & 8 & 7 & 15 \\
\hline SURVIVORS & 5 & 36 & 41 \\
\hline TOTAL & 13 & 43 & 56 \\
\hline
\end{tabular}

HRS associated with higher mortality when compared with non HRS with $\mathrm{P}$ value of 0.004 , which is statistically significant. 
Association of DIALYSIS with NON DIALYSIS in predicting MORTALITY

\begin{tabular}{|l|c|c|c|}
\hline & DIALYSIS & NON DIALYSIS & TOTAL \\
\hline NON SURVIVORS & 9 & 6 & 15 \\
\hline SURVIVORS & 9 & 32 & 41 \\
\hline TOTAL & 18 & 38 & 56 \\
\hline
\end{tabular}

Significant P Value of 0.0001 , indicating that RRT has a significant impact on mortality

\section{Discussion}

The incidence of AKI in patients with cirrhosis of the liver in the present study was $25.8 \%(56 / 217)$; the result is similar to the study by Jai Prakash et $\mathrm{al}^{3}$. where the incidence of AKI was $24.5 \%$.

Incidence of HRS in our study was $23 \%$ of the total cases of AKI, and the majority $(10 / 13 ; 76 \%)$ were of type $1 \mathrm{HRS}$. This is in agreement with the study of Moreau et al. .25, who reported that the incidence of HRS was $20 \%$ in their cirrhotic patients.

A strong step-wise association was noted between the degree of progression of AKI and mortality. AKI in the setting of cirrhosis was ultimately severe with peak AKIN stages 1, 2, and 3 attained in $14 \%, 30 \%$, and $55 \%$ of patients, respectively. The present study is similar to Belcher JM et $\mathrm{al}^{4}$. Mortality increased in a stage response manner with the severity of AKI. In the Present study, high mortality is associated with AKIN stage 3 with significant $\mathrm{P}$ value 0.002 ( $\mathrm{p}<0.05$ ). Remarkably, patients with a peak severity of AKIN stage 1 did exceptionally well. Nonsurvivors ultimately experienced significantly more severe AKI.

Dialysis was required for 18 (32\%) patients and was utilized more frequently among non-survivors with $\mathrm{P}$-value 0.001 , which was statistically significant, similar to Belcher JM et $\mathrm{al}^{4}$.

\section{Conclusion}

The incidence of AKI in patients with cirrhosis of the liver was $25.8 \%(56 / 217)$. The spectrum of AKI was pre-renal $(44.64 \%)$ intrinsic renal (32.04\%) and HRS (23.14\%). Mortality was correlated with the severity of AKI and the need for hemodialysis

\section{References}

1. D’Amico G, Garcia-Tsao G, Pagliaro L. Natural history and prognostic indicators of survival in cirrhosis: a systematic review of 118 studies. J Hepatol 2006; 44:217-231. doi: 10.1016/j.jhep.2005.10.013.

2. du Cheyron D, Bouchet B, Parienti JJ, Ramakers M, Charbonneau P. The attributable mortality of acute renal failure in critically ill patients with liver cirrhosis. Intensive Care Med 2005; 31:1693-1699. doi: 10.1007/s00134-005-2842-7.

3. Jai Prakash, Amit Kumar Mahapatra, Biplab Ghosh, Puneet Arora and Ashok Kumar Jain Clinical Spectrum of Renal Disorders in Patients with Cirrhosis of Liver Renal Failure, 33(1): 40-46, (2011.

4. J. M. Belcher, G. Garcia-Tsao, A. J. Sanyal et al., "Association of AKI With mortality and complications in hospitalized patients with cirrhosis," Hepatology, vol. 57, no. 2, pp. 753-762, 2013. 